\title{
Zukunftspessimismus in der Frühen Neuzeit? Überlegungen zu frühneuzeitlichen Zukunftsvorstellungen am Beispiel des Vanitas-Motives zur Zeit des Barock
}

\author{
Lisa-Marie Gabriel \\ Kerngebiet: Wirtschafts- und Sozialgeschichte \\ eingereicht bei: Univ.-Prof. Dr. Kupper Büchel Patrick \\ eingereicht im Semester: WS 2015/16 \\ Rubrik: BA-Arbeit
}

\begin{abstract}
Future pessimism in the Early Modern era? Reflections on Early Modern future imaginations exemplified by the baroque Vanitas motif

The following bachelor thesis is about the future imaginations of the Early Modern time. The paper follows the question, if there was a factual, future pessimism' in the baroque era, trying to exemplify this by the booming Vanitas motif. Therefore, the thesis will examine the history and meaning of the motif compared to the trends of the tumultous $17^{\text {th }}$ century. As will be shown, the Vanitas motif was also an art form as well as a manifestation of a transienceconsciousness that manifested itself not least in the form of Vanitas still life and baroque poetry.
\end{abstract}

\section{Einleitung}

"Das Zukünftige geht uns nichts an, weder daß es ist, noch daß es nicht ist; wir dürfen keine Unruhe deshalb haben. Dies ist der richtige Gedanke über die Zukunft", schrieb G.W. F. Hegel in den 1820er-Jahren in seiner Geschichte der Philosophie. Eine Prämisse, die zumindest in der Historikerlnnen-Zunft lange anerkannt war, langsam aber aufzubrechen scheint, erfreut sich die Futurologie, also die Lehre von der Zukunft, doch seit einigen Jahren zunehmender Beliebtheit als Forschungsgegenstand - auch in den

Georg Wilhelm Friedrich Hegel, Vorlesungen über die Philosophie der Geschichte, in: Georg Wilhelm Friedrich Hegel, Werke in zwanzig Bänden, Eva Moldenhauer und Karl Markus Michel (Hrsg.), Band 17, Frankfurt am Main 1969, S. 331. 
Geschichtswissenschaften. ${ }^{2}$ Dass gerade letztere dabei ebenso ihren legitimen Platz im Feld der Zukunftsforschung haben wie andere Wissenschaften, zeigt nicht zuletzt eine Definition des Untersuchungsgegenstandes des deutschen Physikers, Soziologen und Zukunftsforschers Rolf Kreibich aus dem Jahr 2006:

"Zukunftsforschung ist die wissenschaftliche Befassung mit möglichen, wünschbaren und wahrscheinlichen Zukunftsentwicklungen und Gestaltungsoptionen sowie deren Voraussetzungen in Vergangenheit und Gegenwart." ${ }^{13}$

Die historische Zukunftsforschung wirkt dabei insbesondere auf dem "Gebiet der historischen Horizontbildung", als auch im Bereich der "Zukunftsgestaltung".5 Das heißt, die Geschichtswissenschaft erforscht ebenso vergangene Zukunftsvorstellungen, als sie auch aus der Vergangenheit Tendenzen zur gegenwärtigen Zukunftsentwicklung abzuleiten versucht - die Geschichte also auch in diesem Feld eine magistra vitae.

Eine Analyse auf dem Gebiet der historischen Horizontbildung ist gewissermaßen auch die hier vorliegende Bachelorarbeit. Deren Ziel ist eine exemplarische Untersuchung vergangener, das heißt frühneuzeitlicher Zukunftsvorstellungen, festgemacht am Beispiel des Vanitas-Motives zur Zeit des Barock. Eine Themenwahl, die aus der Überlegung heraus zustande kam, dass der Epochenbegriff Neuzeit schon seit seiner Prägung durch die Humanisten um 1500 überaus positiv konnotiert war und es auch heute noch oft ist. Er suggeriert das Bild eines geradezu glanzvollen neuen Zeitalters mit ebenso glanzvollen Zukunftsaussichten. Ein Eindruck, der bei genauerer Betrachtung der Epochenentwicklungen - man denke etwa an die verheerenden Folgen der Kleinen Eiszeit, den Dreißigjährigen Krieg, die Inquisition, die Revolutionen, etc. - allerdings nur allzu bald fraglich erscheint. Umso mehr, wenn man den Blick auf das geradezu allgegenwärtige Vanitas-Motiv und die damit verbundene Mahnung an den stets nahenden Tod rund um das 17. Jahrhundert wendet.

Kann für die Zeit des Barock, als Ausschnitt der Frühen Neuzeit, also tatsächlich von einem ausgemachten Zukunftspessimismus gesprochen werden? Und ist diese Idee am Beispiel des Vanitas-Motives festzumachen oder handelt es sich bei diesem vielmehr um eine rein künstlerische Verarbeitung eines gängigen Topos in einer der vielleicht verspieltesten Epochen der europäischen Kunst- und Kulturgeschichte? Was hat es mit den Zukunftsvorstellungen der frühneuzeitlichen Gesellschaft überhaupt auf sich und unter welchen Voraussetzungen ist eine solche Analyse zu betrachten? Dies sind die Fragen, denen sich diese Arbeit widmet. Dabei ist davon auszugehen, dass aufgrund der krisenbehafteten Zeitumstände gerade in der Zeit des Barock eine überaus pessi-

2 Lucian Hölscher, Die Entdeckung der Zukunft (Europäische Geschichte), Frankfurt am Main 1999, S. 9. Siehe auch Alexander Schmidt-Gernig, Rezension zu: Lucian Hölscher, Die Entdeckung der Zukunft, in: H-Soz-Kult 10.02.2000, online [http://hsozkult.geschichte.hu-berlin.de/rezensionen/252.pdf], eingesehen 16.9.2016. Hölscher und sein Rezensent berichten für die Zeit der Jahrtausendwende noch von einer recht zögerlichen Auseinandersetzung der Historikerlnnenzunft mit der Geschichte der Zukunft.

3 Rolf Kreibich, Zukunftsforschung. ArbeitsBericht Nr. 23/2006, IZT, Berlin 2006, S. 3, online [https://www.izt.de/ fileadmin/downloads/pdf/IZT_AB23.pdf], eingesehen 1.10.2015.

$4 \quad$ Hölscher, Entdeckung der Zukunft, S. 234.

5 Ebd., S. 235 
mistische Zukunftsschau aktuell war. In diesem Zusammenhang ist auch zu überlegen, ob das Vanitas-Motiv seinerzeit tatsächlich eine über das künstlerische Interesse hinausgehende Konjunktur erlebte, oder ob dem bekannten Vergänglichkeits-Topos in dieser Zeit keine größere Bedeutung beizumessen ist.

Unter Bezugnahme auf die einschlägige Forschungsliteratur sowie auf drei ausgewählte zeitgenössische Quellen sucht die vorliegende Arbeit in drei größeren Abschnitten Antworten auf die formulierten Fragestellungen und Hypothesen. Ein erster Teil widmet sich dabei zunächst dem vorrangigen Untersuchungsgegenstand: Was heißt überhaupt Zukunft und was ist in Hinblick auf die Analyse vergangener Zukunftsvorstellungen zu beachten? Das darauffolgende Kapitel widmet sich davon ausgehend speziell den Tendenzen frühneuzeitlicher Zukunftsentwürfe: Was lässt sich grundsätzlich zur Konnotation gegenüber dem Begriff Zukunft in dieser Epoche aussagen und welche Entwicklungslinien lassen sich, womöglich speziell für die Zeit des Barock, festmachen? Der dritte und umfangreichste Teil dieser Arbeit beschäftigt sich davon ausgehend explizit mit der Analyse der barocken Zukunftsmentalität am Beispiel des Vanitas-Motives als exemplarischem Auszug frühneuzeitlicher Zukunftsgeschichte. Dabei werden sowohl das Vanitas-Motiv als auch die Epochenhintergründe des Barock genauer in Augenschein genommen, die es anhand der zeitgenössischen Quellen aus den Bereichen Kunst und Literatur kritisch zu hinterfragen gilt.

Was den Literatur- und Forschungsstand zur hier gewählten Thematik anbelangt, so wurde bereits eingangs darauf verwiesen, dass die Zukunftsforschung gerade in den Geschichtswissenschaften lange Zeit stiefmütterlich behandelt wurde. Dennoch finden sich mehrere hilfreiche Publikationen sowohl aus den Prä- als auch aus den Post2000er-Jahren. Als Referenzwerk zum Aspekt der Zukunftsvorstellung ist dabei zweifellos Lucian Hölschers 1999 erschienene Monographie „Entdeckung der Zukunft” zu nennen. Hilfreich waren weiterhin „Zeit und Kultur. Geschichte des Zeitbewußtseins in Europa“ aus dem Jahre 1980 von Rudolf Wendorff, sowie der 2004 veröffentlichte Titel "Zeitkulturen“ von Lutz Götze. Weiterführend waren auch Beiträge der Enzyklopädie der Neuzeit zu diversen Aspekten der vorliegenden Thematik. So etwa der Eintrag Zeiterfahrung von Eric-Oliver Mader (einen eigenen Beitrag unter dem Schlagwort Zukunft oder einem ähnlichen Terminus führt das Nachschlagewerk nicht), sowie die Einträge Barock und Vanitas, jeweils von Kennern der Materie bearbeitet. Letztere konnten von einem verhältnismäßig umfangreicheren Literaturangebot, als dies in Bezug auf (frühneuzeitliche) Zukunftsvorstellungen der Fall ist, ergänzt werden. So etwa von diversen Kompendien zu barocker Kunst und Literatur, wie Sabine Burbaums Kompaktdarstellung zur Kunst-Epoche Barock, erschienen 2003, oder dem ersten Band „Deutsche Literaturgeschichte" des Forscherpaares Erika und Ernst von Borries in der Auflage von 2006. 


\section{Zukunftsvorstellung: Zu Begriff und Problematik des Untersuchungsgegenstandes}

Will man sich mit vergangenen Zukunftsvorstellungen beschäftigen, fragt man nicht allein nach dem Begriff der Zukunft und seiner jeweiligen Bedeutung, sondern man beschäftigt sich zwangsläufig auch mit der Zeit. Ein Umstand, der ein ungleich weiteres Begriffsfeld eröffnet: Die Rede ist von der Zeitordnung und Zeitrechnung, von der Zeitwahrnehmung, vom Zeitempfinden und Zeitbewusstsein. Lutz Götze, der sich in einer 2004 erschienenen Studie mit Zeit in verschiedenen Kulturen der Erde sowie in unterschiedlichen wissenschaftlichen Feldern auseinandergesetzt hat, spricht etwa von der Linearität im Gegensatz zur Zirkularität des Zeitbewusstseins und von der absoluten im Gegensatz zur subjektiv erlebten Zeit. ${ }^{6}$ All diese genannten Aspekte sind Möglichkeiten zur Annäherung an den Umgang mit dem Phänomen Zeit, das in einer Gesellschaft und innerhalb dieser selbst von den einzelnen Mitgliedern mitunter jeweils ganz unterschiedlich aufgefasst wird. Das heißt, die Zeit - und damit auch das Verständnis von "Vergangenheit", "Gegenwart" und Zukunft - ist jeweils subjektiv sowie kulturell bedingt. Oder wie Götze in Entlehnung der Worte Isaac Newtons meint: „Zeit ist, Menschenwerk.".7

Wenn diese Aspekte für die Zeit als solche gelten, müssen sie demnach ebenso für die Zukunft - sozusagen als Ausschnitt der Zeit - geltend gemacht werden. Tatsächlich ist auch die Zukunft ein reines Gedankenkonstrukt, ein Produkt erschaffen von Menschenhand, das je nach räumlichen, kulturellen und selbst individuellen Bedingungen unterschiedlich erlebt und erfahren wird. Alleine aus diesem Grund scheint es auch sinnvoll, von Zukünften anstelle von Zukunft zu sprechen. Nicht zuletzt da es quer durch die Epochen betrachtet eine ausgesprochene Fülle an Zukunftsvorstellungen gab, „die zu verschiedenen Zeiten, bei verschiedenen Menschen und hinsichtlich verschiedener Gegenstände bestanden haben"8. Zukunftsvorstellungen sind letztlich also mentalitätsgeschichtliche Konstrukte, die als solche mitunter nur schwer zu fassen sind und deren Betrachtung durchaus nicht frei von Problemen ist, etwa was die Quellen anbelangt, die oft nicht oder nur unzureichend vorhanden sind, nur eine bestimmte Gesellschaftsschicht oder Weltsicht abbilden und so weiter. ${ }^{9}$ Erschwerend hinzu kommt, dass die Erforschung der Zukunft, die grundsätzlich interdisziplinär zu bearbeiten und zudem kein "fest umrissene Wissenschaft" ${ }^{\prime 10}$ ist, ${ }^{11}$ von Wissenschaftlerlnnen ganz unterschiedlich bewertet wird.

6 Lutz Götze, Zeitkulturen. Gedanken über die Zeit in den Kulturen (Im Medium fremder Sprachen und Kulturen 6), Frankfurt am Main 2004, S. 10; Eric-Oliver Mader, Zeiterfahrung, in: Enzyklopädie der Neuzeit, online [http://referenceworks.brillonline.com/entries/enzyklopaedie-der-neuzeit/zeiterfahrung-a4858000], eingesehen 4.11.2015.

7 Götze, Zeitkulturen, S. 10; Ebd., S. 9 -10.

8 Hölscher, Entdeckung der Zukunft, S. 10.

9 Hierzu ausführlicher Peter Dinzelbacher, Zu Theorie und Praxis der Mentalitätsgeschichte, in: Peter Dinzelbacher (Hrsg.), Europäische Mentalitätsgeschichte. Hauptthemen in Einzeldarstellungen, Stuttgart 1993, S. XV-XXXVII.

10 Kreibich, Zukunftsforschung, S. 3.

11 Ebd., S. 3-4. 
Während etwa Rolf Kreibich von den Möglichkeiten der Zukunftsforschung berichtet und von vornherein von Zukünften ${ }^{12}$ als Forschungsgegenstand spricht, meint im Gegensatz dazu beispielsweise die Historikerin und Philosophin Doris Gerber, dass es unabhängig vom Betrachtungszeitpunkt jeweils nur eine offene Zukunft geben kann. Die Wissenschaftlerin, die sich kritisch mit dem von Reinhart Koselleck weithin tradierten Begriff von der "vergangenen Zukunft" beschäftigt hat, ${ }^{13}$ geht sogar so weit zu sagen, dass jeder "Versuch, irgendeine bestimmte Vergangenheit als eine auf ihre jeweilige Zukunft verwiesene zu untersuchen, [...] misslingen [muss]",14 da die Geschichtswissenschaft ihre Analysen zwangsläufig nur auf die einzig vorhandene, nämlich die eigene Zukunft beziehen kann. ${ }^{15}$ Ein Umstand, den durchaus auch Hölscher in seinem bereits 1999 erschienenen Werk erkannt hat. Weniger ausschließend als etwa Gerber leitet er aus dieser Schwierigkeit die Notwendigkeit eines erhöhten Abstraktionsvermögens des Betrachtenden "gegenüber dem gewandelten historischen Kontext ${ }^{116}$ ab. ${ }^{17}$

So weit zu den konstitutiven Elementen des Betrachtungsgegenstandes. Was lässt sich nun aber konkret zur Zukunft und ihren jeweiligen Vorstellungen festhalten? Definiert wird Zukunft als "die Zeit, die noch bevorsteht, die noch nicht da ist: die erst kommende oder künftige Zeit (und das in ihr zu Erwartende)"18. Ein eigentlich neutraler Ausdruck, der durch eben diese Erwartungshaltung seinen ganz eigenen Charakter erhält. So lässt sich der Begriff etymologisch betrachtet zunächst in Form des Adjektivs zukünftig, über das mittelhochdeutsche zuokunft und das althochdeutsche zuokumft bis in das 9. Jahrhundert zurückverfolgen, was seinerzeit so viel bedeutete wie "das Herankommende"19. Eine Bedeutung, die den Eindruck von Unausweichlichkeit und Unabänderlichkeit erweckt, von dem was da nicht nur mit größter Wahrscheinlichkeit, sondern scheinbar sicher herannaht. Eine andere Bedeutungsperspektive eröffnet hingegen die Begriffsdefinition laut Duden. So vermitteln die Synonyme "Aussicht, Chance, Hoffnung, Möglichkeit [...]"20 allesamt den Eindruck einer offenen, teils gestaltbaren und grundsätzlich positiven Zukunftshaltung, die ganz im Sinne einer "es wird schon alles (gut) werden"-Mentalität zu stehen scheint. Ähnliches führt auch Hölscher im Zusammenhang weiter aus:

„[...] der Begriff der ,Zukunft [ist] schon immer doppeldeutig gewesen [...], indem er zwei äußerst heterogene, ja geradezu widersprüchliche Vorstellungen

12 Kreibich, Zukunftsforschung S. 3-4.

13 Reinhart Koselleck, Vergangene Zukunft. Zur Semantik geschichtlicher Zeiten (suhrkamp taschenbuch wissenschaft 757), Frankfurt am Main 1979.

14 Doris Gerber, Was heißt, vergangene Zukunft'? Über die zeitliche Dimension der Geschichte und die geschichtliche Dimension der Zeit, in: Geschichte und Gesellschaft 32 (2006), Heft 2, S. 176-200, hier S. 178.

15 Gerber, Dimension der Zeit, S. 177-178.

16 Hölscher, Entdeckung der Zukunft, S. 235.

17 Ebd., S. 234-235.

18 Zukunft, in: Duden, online [http://www.duden.de/rechtschreibung/Zukunft], eingesehen 7.10.2015.

19 Zukunft, in: Friedrich Kluge, Etymologisches Wörterbuch der deutschen Sprache, Berlin-New York, 2012 ${ }^{25}$, online [https://vpn.uibk.ac.at/+CSCO+1h756767633A2F2F6A6A6A2E71727465686C6772652E70627A++/view/Kluge/ kluge.12813?rskey=0fC8GB\&result=1\&dbq_0=zukunft\&dbf_0=kluge-fulltext\&dbt_0=fulltext\&o_0=AND], eingesehen 7.10.2015. Der zugehörige Substantiv,Zukunft' lässt sich in dieser Bedeutung interessanterweise vereinzelt erst im Spätmittelalter nachweisen. Ebd. 
miteinander verknüpft: einerseits die Vorstellung, dass sich die Dinge, die wir erwarten, aus der Geschichte und Gegenwart ableiten lassen, andererseits die gerade umgekehrte Vorstellung, daß diese, wie schon das Wort selbst sagt, auf uns, zukommen.".'21

Zukunft kann also gar nicht mehr als ein Gedankenkonstrukt, eine Idee sein. Zwar lassen sich mitunter Wahrscheinlichkeiten ableiten, etwa in Hinblick auf das morgige Wetter, und es bilden sich - zumeist ganz unbewusst - Erwartungshaltungen an das Künftige, die sich im Nachhinein denn auch bestätigen mögen. Dennoch ist die Zukunft von ihrem grundsätzlichen Wesen her schlichtweg nicht oder nur sehr eingeschränkt - beispielsweise folgen die Jahreszeiten weiter aufeinander - bekannt.22

Dies ist wohl nicht zuletzt auch der Grund für die Entwicklung von Prophetie und Prognostik, Instrumente, mit denen die Menschen zu allen möglichen Zeiten dieser Unbekannten namens Zukunft zu begegnen suchten. Ein Bedürfnis, das etwa der französische Historiker Georges Minois als "Eigentümlichkeit des Menschen"23, als "grundlegende Dimension seines Daseins"24 ansieht. Dennoch wäre es fatal, den Wunsch danach, die Zukunft zu kennen, als anthropologische Konstante beschreiben zu wollen. „[D]ie Fähigkeit, sich selbst in eine Zukunft hinein zu entwerfen, [ist ...] keine Vorgegebenheit menschlicher Existenz schlechthin [...], sondern eine historisch spezifische Denkform"25, insistiert im Zusammenhang etwa Hölscher. Welche Denkformen dahingehend in der Frühen Neuzeit vorherrschten und wie sich diese speziell zur Zeit des Barock äußerten, ist Inhalt der beiden folgenden Kapitel.

\section{Tendenzen frühneuzeitlicher Zukunftsvorstellungen}

Entsprechenden Darstellungen wie etwa Rudolf Wendorffs „Zeit und Kultur. Geschichte des Zeitbewußtseins in Europa"26 zufolge, lassen sich wenigstens seit der Zeit der Frühen Hochkulturen spezifische Zeit- beziehungsweise auch Zukunftsvorstellungen nachvollziehen. Wendorff führt beispielsweise in vierzehn Kapiteln, angefangen im alten Babylonien über die Kulturen der klassischen Antike und das Frühe Christentum, quer durch das Zeitbewusstsein der europäischen Kulturepochen bis hinein in das 20. Jahrhundert. ${ }^{27}$ Das heißt, sowohl die Zeit(-rechnung) als auch die (Vorstellungen von der) Zukunft haben eine Geschichte, ${ }^{28}$ und beide erfuhren gerade im Zuge der Frühen

21 Hölscher, Entdeckung der Zukunft, S. 12.

22 Georges Minois, Geschichte der Zukunft. Orakel, Prophezeiungen, Utopien, Prognosen, Düsseldorf-Zürich 1998, S. 17. Der deutsche Historiker Dieter Geuenich sieht als einzige bekannte Zukunft tatsächlich sogar nur den Tod an: „Dieses Ende, der Tod, ist die Zukunft jedes Menschen; der Tod ist also das, was auf jeden Menschen unaufhaltsam,zukommt', ist seine Zukunft schlechthin. "Dieter Geuenich, Zukunftsvorstellungen im Mittelalter. Antrittsvorlesung an der Universität - GH - Duisburg am 30. Mai 1989, online [http://www.univie.ac.at/igl.geschichte/zeller/ ws_2010_11/materialien_ku/Geuenich.pdf], eingesehen 16.11.2015.

23 Minois, Geschichte der Zukunft, S. 17.

24 Ebd

25 Hölscher, Entdeckung der Zukunft, S. 10.

26 Rudolf Wendorff, Zeit und Kultur. Geschichte des Zeitbewußtseins in Europa, Opladen 1980.

27 Wendorff, Zeit und Kultur.

28 Darauf deutet nicht zuletzt etwa Georges Minois' umfangreiche Publikation mit dem Titel "Geschichte der Zukunft" aus dem Jahr 1998 hin. 
Neuzeit eine ausgesprochene Wende. Dies soll freilich nicht darüber hinwegtäuschen, dass sich auch die vorhergehenden Epochen und Zeitabschnitte in ihren Vorstellungen von der Zeit im Allgemeinen und der Zukunft im Speziellen teils empfindlich unterschieden haben. ${ }^{29}$ Dennoch markieren die dahingehenden Entwicklungen im Zuge der Frühen Neuzeit eine einschneidende Zäsur, die etwa Lucian Hölscher von der „Entdeckung der Zukunft" ${ }^{\prime \prime 30}$ sprechen lassen.

So etablierten sich zwar schon im Frühen Mittelalter nach und nach eindeutiger festzumachende Zeit- und Zukunftsvorstellungen, die mit der Erfindung der ersten Räderuhren mehr und mehr der modernen Zeitgliederung entsprachen. ${ }^{31}$ Der Zeit- und Zukunftshorizont des Mittelalters war allerdings noch ein geschlossener" Er war durch das eschatologische Welt- und Zeitverständnis auf ein Ende hin, auf das "Endgericht“32 nämlich, ausgerichtet. ${ }^{33} \mathrm{Neu}$ an der Neuzeit war demnach nicht die Entdeckung"vom Zukünftigen überhaupt, sondern vielmehr die Entdeckung der Zukunft, als einer "homogenen, allmählich verfließenden Zeit"34, als ", einheitlichem geschichtlichem Zeitraum"35, der nicht mehr auf ein scheinbar bekanntes Ende hin ausgerichtet war, sondern Möglichkeiten zur Realisierung und Handlung bot. Die Zukunft wurde somit offen und weit. Ein Umstand, der ebenso die positiv konnotierte Möglichkeit zur eigenen Zukunftsgestaltung beinhaltete, zugleich aber auch eine regelrechte Angst vor der offenen und daher unbekannten und unberechenbarer gewordenen Zukunft barg. ${ }^{36}$

Dazu muss allerdings erwähnt werden, dass diese Entwicklung vom geschlossenen zum offenen Zukunftshorizont ein langwieriger Prozess war, wenngleich er aus heutiger Sicht tatsächlich als Einschnitt im abendländischen Zeit- respektive Zukunftsbewusstsein betrachtet werden kann. So versteht beispielsweise Rudolf Wendorff das Spätmittelalter in dieser Hinsicht als „Übergangsfeld“37 und konstatiert spätestens für das 16. Jahrhundert einen grundlegenden Wandel im Hinblick auf das abendländische Zeitbewusstsein. ${ }^{38}$ Auch Lucian Hölscher stellt einen dahingehenden Perspektivenwechsel für das westliche Europa zur Zeit des Dreißigjährigen Krieges fest, wenngleich er an anderer Stelle darauf verweist, dass die "eschatologische Endzeiterwartung die westeuropäische Gesellschaft selbst in ihren gebildeten, akademischen Schichten"39

29 „Bis zum Ende des Mittelalters kann man bei den verschiedenen Kulturen von einem jeweils vorherrschenden Zeitbewußtsein sprechen und diese Signatur in den unterschiedlichsten Lebensbereichen gleichzeitig bzw. hier und da mit leichten Verzögerungen erkennen", führt beispielsweise Wendorff im Zusammenhang näher aus. Wendorff., Zeit und Kultur, S. 151.

30 So der Titel der gleichnamigen Monographie, in der sich Hölscher mit der Geschichte der Zukunft vom Mittelalter bis in die 2000er-Jahre beschäftigt, mit Fokus auf die „Entstehung des modernen Zukunftsbegriffs“ (S. 34) seit der Frühen Neuzeit. Hölscher, Entdeckung der Zukunft, 1999. Wendorff, Zeit und Kultur, S. 92-112, S. 129-130, S. 134-136, S. 147-150.

Hölscher, Entdeckung der Zukunft, S. 23.

Ebd., S. 17-34; Geuenich, Zukunftsvorstellungen im Mittelalter, online.

Hölscher, Entdeckung der Zukunft, S. 9.

Ebd., S. 9, S. 28.

Niklas Luhmann, The Future Cannot Begin: Temporal Structures in Modern Society, in: Social Research, 43 (1976), Nr. 1, S. 130-152, hier S. 130-132; Hölscher, Entdeckung der Zukunft, S. 23-33. 
noch bis ins 17. Jahrhundert hinein beherrschte. ${ }^{40}$ Der Wandel vom geschlossenen endzeitlichen zum offenen, selbst gestaltbaren, dadurch aber auch Unsicherheit bergenden Zukunftshorizont der (Frühen) Neuzeit vollzog sich letztlich erst im Verlauf des 17. und 18. Jahrhunderts. Niklas Luhmann nennt als letztmöglichen Zeitpunkt hinsichtlich eines gewandelten abendländischen Zukunftsverständnisses jedenfalls die Zeit der Französischen Revolution. ${ }^{41}$

Hauptgrund dafür war wohl vor allem die Tatsache, dass sich die Idee vom Weltenende aus unterschiedlichen Überlegungen heraus nicht mehr tragen ließ. Eine zunehmende Unsicherheit gegenüber dem aus der Bibel errechneten Alter der Welt ließ den scheinbar so nahen Zeitpunkt vom Weltenende zunehmend unglaubwürdig erscheinen. Die weitreichende und bis in unsere Zeit hineinreichende Konsequenz war nicht nur die Einführung einer neuen Zeitrechnung (die Zeit vor und nach dem neu errechneten Nullpunkt wurde jeweils mit dem Zusatz vor beziehungsweise nach Christi Geburt versehen), sondern auch ein neuer Geschichtsbegriff, der die weitere Entwicklung des neuzeitlich-modernen Zukunftsverständnisses entschieden beeinflusste, wie Doris Gerber etwa wie folgt zusammenfasst:42

„Zum einen ist der neuzeitliche Geschichtsbegriff durch die Ablösung christlicher Endzeitvorstellungen geprägt. Erst nach der Hinfälligkeit solcher eschatologischer Prophezeiungen kann Geschichte als etwas begriffen werden, das in eine offene und prinzipiell unendliche Zukunft hinein verläuft. Diese Vorstellung einer unendlichen Offenheit ist die Voraussetzung für die spekulativen Geschichtsphilosophien des 18. und 19. Jahrhunderts, die unter anderem den Versuch darstellen, die mit dieser Vorstellung verbundene Ungewissheit dadurch zu beruhigen, dass die Geschichte als ein a priori vorbestimmter Prozess einer stetigen Realisierung der Idee der Vernunft beschrieben wird. Noch im Laufe des 19. Jahrhunderts differenziert sich dann dieser ursprünglich alles umklammernde Vernunftglaube in viele unterschiedliche und politisch konkretisierte Fortschrittsideen aus." ${ }^{43}$

Damit sind nun die wichtigsten Entwicklungslinien des frühneuzeitlichen Zukunftsverständnisses inklusive ihrer langfristigen Auswirkungen abgesteckt. Zur weiteren Annäherung an mögliche Zukunftsvorstellungen der Frühen Neuzeit dient die nachfolgende Analyse der barocken Zukunftsmentalität am Beispiel des Vanitas-Motives.

\section{Zukunftsvorstellungen im Barock am Beispiel des Vanitas-Motives}

Wie eben dargelegt, war die (Frühe) Neuzeit selbst im Hinblick auf das Zeit- und Zukunftsbewusstsein im (westlichen) Europa gewissermaßen eine Zeit der Entdeckung. Allerdings ist in diesem Kontext insbesondere die Untersuchung des barocken Zu-

\footnotetext{
40 Hölscher, Entdeckung der Zukunft, S. 30-31.

41 Luhmann, Future cannot begin, S. 130-132.

42 Gerber, Dimension der Zeit, S. 176-177; Hölscher, Entdeckung der Zukunft, S. 31-34; Luhmann, Future cannot begin, S. 130-132.

43 Gerber, Dimension der Zeit, S. 177.
} 
kunftsverständnisses ambivalent zu betrachten, da sich die Teilepoche geradewegs an der Schnittstelle zwischen dem geschlossenen, eschatologisch bestimmten Zukunftsglauben des Mittelalters, der noch bis in das 17. Jahrhundert hineinwirkte, und dem neuen, offenen Zukunftshorizont, der letztlich die Neuzeit prägte, bewegt. Hinzu kommt, dass dieses neue Zukunftsverständnis gewissermaßen aus einer tiefschürfenden Erfahrung der Unsicherheit in Hinblick auf die biblische Zeitrechnung erwachsen ist. Welche Ausformung die barocke Zukunftssicht unter diesen Voraussetzungen angenommen hat, ist Gegenstand der nun folgenden Ausführungen.

\subsection{Zum Motiv der Vanitas}

Der Begriff Vanitas stammt aus dem Lateinischen und bedeutet so viel wie Leere, falscher Schein, Prahlerei, Vergänglichkeit. ${ }^{44}$ Im Wesentlichen handelt es sich bei der Vanitas also um ein Motiv oder Thema der bildenden Kunst, Literatur und Musik, das für die Vergänglichkeit alles Irdischen steht. Eine Symbolik, die sich bereits im Alten Orient, im Alten Ägypten sowie in den Kulturen der klassischen Antike findet. Gleichwohl gründet sich die abendländische Vanitas-Tradition der Nach-Antike weniger auf diese antiken Vorläufer als vielmehr auf die Idee der vanitas mundi, also die Idee von der Vergänglichkeit der Welt, die über die christliche Theologie vermittelt wurde. So kam es gerade zur Zeit der Kirchenväter im 4. beziehungsweise 5. Jahrhundert nach Christus zu einer Radikalisierung dieser These, die denn auch die nachfolgenden Epochen bestimmte: Als Ursache für die Endlichkeit der menschlichen Welt wurde der biblische Sündenfall durch das menschliche Erkenntnisstreben angesehen, woraus die Forderung entstand, sich auf das jenseitige, unendliche Leben fern der irdischen Vergänglichkeit zu orientieren. $^{45}$

Womöglich ist dies die Erklärung für die stark heilsgeschichtliche Ausrichtung der mittelalterlichen Zukunftsmentalität, die vom sicheren Kommen des letzten Zeitalters dem Reich Gottes - ausging, für das es sich zu rüsten galt. Interessanterweise hatte das Vanitas-Motiv aber auch in den Jahrhunderten der Frühen Neuzeit - einer Zeit des Fortschritt- und Erkenntnisstrebens - Konjunktur. Ein Umstand, der womöglich die Idee vom Sündenfall durch das unangebrachte menschliche Erkenntnisinteresse, das gewissermaßen das Hinterfragen der göttlichen Schöpfung bedeutete, neu belebte. So gesehen, hatte das Motiv der Vanitas also zu unterschiedlichen Zeiten und aus unterschiedlichen Beweggründen Hochzeit. Die konkrete Darstellungsform der Vanitas in Kunst und Literatur war dabei jeweils abhängig vom historischen Kontext. Eine erste Blüte erlebte der Topos demnach mit dem Aufkommen der mittelalterlichen Vergänglichkeitsdichtung und -malerei im 12. Jahrhundert, in deren Zuge auch eine „erste weitreichende Popularisierung der Vanitas-Idee"46 entstand. Die entsprechenden Ausdrucksformen beschränkten sich seinerzeit aber noch auf allegorische Darstellun-

44 Jens Ewen, Vanitas, in: Enzyklopädie der Neuzeit, online [http://referenceworks.brillonline.com/entries/enzyklopaedie-der-neuzeit/vanitas-a4511000], eingesehen 19.11.2015. Vanitas, in: Duden, online [http://www. duden.de/rechtschreibung/Vanitas], 19.11.2015.

45 Ewen, Vanitas, ENZ online.

46 Ebd. 
gen wie die der Frau Welt oder auf die sogenannten Totentänze, differenzierten sich im Zuge des 14. und 15. Jahrhunderts aber nach und nach aus. In dieser Zeit erhielten die einzelnen Symbole, wie etwa der Totenkopf, ihre für lange Zeit gültigen Konnotationen. ${ }^{47}$

Einen neuerlichen Aufschwung erfuhr die Vergänglichkeits-Symbolik schließlich ab Mitte des 16. Jahrhunderts und war Ausdruck des Krisencharakters jener Zeit, wie im nachfolgenden Teilkapitel näher ausgeführt. So avancierten Vanitas-Darstellungen etwa in der Malerei ab circa 1550 zu einem eigenständigen und weit verbreiteten Genre, das vor allem das niederländische Stillleben stark beeinflusste. Standardisierte Symbole wie Totenschädel, Sanduhr, das leere Glas, Spiegel, aber auch Luxusgegenstände und Machtinsignien wie Krone und Zepter sowie florale Motive, etwa Blumen, Blätter und Früchte, ${ }^{48}$ sollten „in moralisierender Absicht an die Vergänglichkeit und Nichtigkeit der Schönheit und der menschlichen Existent gemahnen".49

Genauso wie in der Malerei war auch die Fülle an literarischen Vanitas-Darstellungen gerade zur Zeit des Barock weitaus umfangreicher als etwa noch im Mittelalter. „Das Thema der vanitas (Eitelkeit) haben fast alle Barockdichter aufgegriffen", 50 schreiben hierzu etwa Erika und Ernst von Borries. Einer der bekanntesten Vertreter in diesem Zusammenhang ist Andreas Gryphius, dessen Werk auszugsweise auch im Zuge der exemplarischen Quellenanalyse zum vorliegenden Arbeitsthema vertreten ist. Dazu ist zu erwähnen, dass der Barock denn auch die letzte große Zeit der Vanitas-Darstellungen war. Ein umfangreiches Aufgreifen der irdischen Vergänglichkeitsmotivik verlor durch die zunehmende Säkularisierung der abendländischen Gesellschaft spätestens im Zuge der Aufklärung zusehends an Präsenz, wenngleich das Motiv bis heute nie ganz aus der Kunstgeschichte verschwunden ist. ${ }^{51}$ Warum die Vanitas-Idee ausgerechnet zur Zeit des Barock ihre größte Rezeption erfuhr, ist Inhalt der anschließenden Ausführungen.

\subsection{Zum Barock und den soziokulturellen Hintergründen barocker Vanitas-Darstellun- gen}

Nahezu jedes Werk, das sich dem Barock widmet, nähert sich zunächst dem Begriff und dem zeitlichen Umfang dieser Teilepoche der Neuzeit an. Und obgleich der respektive das Barock die "letzte, alle Gattungen umfassende Epoche der europäischen Kunst"52 war und hinsichtlich ihrer "spezifischen ästhetischen und kulturellen Merkmale[n] in

47 Ewen, Vanitas, ENZ online. Der Autor führt hierzu weiter aus: „In der Renaissance versuchte man, mit VanitasDarstellungen eine stärkere Orientierung an rational nachvollziehbaren Weltdeutungen und die Aufwertung der vorchristlichen Antike zu rechtfertigen. Zugleich wurde aber auch der menschlichen Eitelkeit ein buchstäblicher Spiegel vorgehalten: So finden sich auf zahlreichen frühneuzeitlichen Porträts ebenso wie auf ihrer Rückseite einfache Vanitas-Symbole, darunter häufig Totenköpfe."

48 Zu den wichtigsten literarischen Stilmitteln der Vanitas, gerade in der Barockdichtung: Ferdinand van Ingen, Vanitas und Memento mori in der deutschen Barocklyrik, Groningen 1966, S. 145-266.

49 Ewen, Vanitas, ENZ online; Philippe Ariès, Geschichte des Todes, München 1995', S. 412.

50 Erika von Borries/Ernst von Borries, Deutsche Literaturgeschichte. Band 1. Mittelalter - Humanismus - Reformationszeit - Barock, München 200655, S. 359.

51 Ewen, Vanitas, ENZ online.

52 Sabine Burbaum, Barock (Kunst-Epochen 8; Reclams Universal-Bibliothek 18175), Stuttgart 2003, S. 11. 
den Bereichen Kunst, Literatur und Musik"53 wissenschaftliche Übereinkunft herrscht, differieren die zeitlichen Schranken je nach Betrachtungsrahmen etwas. Prinzipiell wird der Barock, ein noch bis ins späte 19. Jahrhundert hinein überaus negativ konnotierter Begriff, der mit der Vorstellung des Skurrilen und Bizarren, des Schwülstigen, Überladenen und Pompösen, des Künstlichen beziehungsweise Verkünstelten und Verschnörkelten verbunden ist, mit der Zeit vom späten 16. Jahrhundert (teils schon ab etwa 1550) bis zum späten 18. Jahrhundert anberaumt.54 In eben diesen Zeitraum fällt auch die Hochkonjunktur des Vanitas-Motives, wie die vorhergehenden Abschnitte kenntlich gemacht haben, was die Frage aufwirft, warum dem so war: Warum wurde ein Motiv, das Pate steht für die Allgegenwart von der Endlichkeit des irdischen Lebens, ausgerechnet in einer der vielleicht verspieltesten Epochen des Abendlandes so stark rezipiert?

Eine eindrückliche Antwort auf diese Frage findet sich etwa in den Ausführungen Philippe Ariès.' Dem französischen Historiker der Annales-Schule zufolge brachten das 16., 17. und auch noch das 18. Jahrhundert „[...] mit größerer Überzeugtheit als ehemals, den traditionellen Glauben an die Hinfälligkeit des menschlichen Lebens und an die Verweslichkeit des Leibes zum Ausdruck ${ }^{\prime \prime 5}$. Der Autor spricht weiterhin von einem „verkrampfte[n] Nichtigkeitsgefühl“56 jener Zeit, „dem Entlastung zu verschaffen auch der Jenseitshoffnung nicht [gelang] "57, wie dies etwa noch im Mittelalter der Fall gewesen war. Das heißt, anders als beispielsweise im Mittelalter, wurde die Endlichkeit des irdischen Lebens in der barocken Gesellschaft nicht von der tröstenden Vorstellung eines sorgenfreien Lebens im Jenseits überlagert, sondern die Nichtigkeit aller Dinge war vielmehr die allgegenwärtige und niederschmetternde Zukunftsaussicht: „Im Laufe des gesamten 17. und 18. Jahrhunderts treibt die Gesellschaft an steil abschüssigem Hang den Schlünden des Nichts entgegen"58, beschreibt Ariès die Zeitumstände weiter.

Grund für diese pessimistische Weltsicht war der ausgesprochene Krisencharakter der Epoche, der gerade im 17. Jahrhundert - einem Zeitraum den der Barock unabhängig von seiner jeweiligen zeitlichen Begrenzung im Ganzen umfasst - seinen Höhepunkt erreichte. Zumindest wird ein solcher in der Forschung, wenngleich auf ganz unterschiedliche Weise, diskutiert; von einigen Wissenschaftlerlnnen auch negiert. Debattiert wird etwa eine allgemeine Krise des Feudalismus, eine Krise der Frömmigkeit sowie einzeln oder auch in Kombination betrachtet Krisensymptome der "ökonomischen, sozialen und staatlichen Sphäre, in den internationalen Beziehungen sowie in Kunst und Wissenschaft" ${ }^{\text {"59 }}$. Diese führten in manchen Regionen Europas beispielsweise

53 Ulrich Pfisterer, Barock. 1. Einleitung, in: Enzyklopädie der Neuzeit, online [http://referenceworks.brillonline.com/ entries/enzyklopaedie-der-neuzeit/barock-a0349000], eingesehen 25.11.2015.

54 Pfisterer, Barock, ENZ online; Burbaum, Barock, S. 9-11; Hermann Bauer, Barock. Kunst einer Epoche, Berlin 1992, S. 9-10.

55 Ariès, Geschichte des Todes, S. 412.

56 Ebd.

57 Ebd.

58 Ebd.

59 Günter Vogler, Europas Aufbruch in die Neuzeit 1500-1650 (Handbuch der Geschichte Europas 5; UTB 2385), Stuttgart 2005, S. 413. 
zu Bürgerkrieg oder Bauernbewegungen und kulminierten nicht zuletzt im oder auch aus dem Dreißigjährigen Krieg, einem der verheerendsten Ereignisse der gesamten Neuzeit. ${ }^{60}$ Günter Vogler konstatiert im Zusammenhang jedenfalls, dass unabhängig von Ansatz und Argumentation in jedem Fall eine "krisenhafte Situation"61 für das 17. Jahrhundert festzuhalten ist. 62

Die Kunsthistorikerin Sabine Burbaum stellt den Krisencharakter der barocken Epoche beispielsweise als eine von "Wandlungsprozessen"63, von "Gegensätzen und Spannungsfeldern"64 geprägte Zeit dar. ${ }^{65}$ Jens Ewen führt den neuerlichen Aufschwung von Vanitas-Darstellungen seit Mitte des 16. Jahrhunderts hingegen auf eine „umfassende Erfahrung von Unsicherheit"66 zurück, die aus dem geballten Krisenpotenzial der Zeit, angefangen bei den Religionskriegen, aber auch den klimatischen Auswirkungen der Kleinen Eiszeit, Hungersnöten, Krankheitswellen sowie einer neuerlichen Erschütterung des philosophischen und wissenschaftlichen Weltbildes kulminierte. ${ }^{67}$ Oder wie Herbert Lüthy schon 1968 die Zeitumstände so eindrücklich schilderte:

„Die Epoche der Kirchenspaltung und der Religionskriege, das grausame Verenden des erasmischen Humanismus zwischen den Mühlsteinen der konfessionellen Fanatismen und die politische und soziale, von Hexensabbat und apokalyptischer Panik geschüttelte Katastrophenzeit des 17. Jahrhunderts war nicht dazu angetan, einen Geschichtsoptimismus hervorzubringen; die Geschichtsschau des Barock ist die Geschichte als Trauerspiel." ${ }^{68}$

Die Zeit des Barock und gerade das 17. Jahrhundert waren bestimmt von Angst und Unsicherheit. Dass sich die Zahl der Todesfälle vom 16. zum 17. Jahrhundert aufgrund des Dreißigjährigen Krieges vervierfachte - „eine Steigerung, die im Hinblick auf die Relation zur Bevölkerungsvermehrung in den kommenden zwei Jahrhunderten nicht übertroffen wird"69 -, dürfte sein Übriges zur vergänglichkeitsbewussten Zukunftssicht der barocken Gesellschaft beigetragen haben. ${ }^{70}$

Das Vanitas-Motiv kann also tatsächlich als Ausdruck einer überaus pessimistischen Welt- beziehungsweise Zukunftssicht innerhalb des barocken Zeitalters verstanden werden. Denn „[g]eradezu symptomatisch werden in Krisenzeiten vermehrt Zukunfts-

60 Günter Vogler, Europas Aufbruch in die Neuzeit 1500-1650 (Handbuch der Geschichte Europas 5; UTB 2385), Stuttgart 2005, S. 413-416.

61 Ebd., S. 416

62 Ebd., S. 413-416.

63 Burbaum, Barock, S. 11.

64 Ebd

65 Burbaum führt hinsichtlich des krisengeprägten 17. Jahrhunderts das Folgende näher aus: „Das 17. Jahrhundert in Europa war geprägt von Wandlungsprozessen, die die politischen Strukturen dauerhaft veränderten, das gesellschaftliche Leben beeinflussten und sich in Gegensätzen und Spannungsfeldern äußerten. Verheerende Kriege, politische Instabilität und soziale Not der Bevölkerung standen Wohlstand und immense Prachtentfaltung fürstlicher Höfe gegenüber [sic.]." Burbaum, Barock, S. 11.

66 Ewen, Vanitas, ENZ online.

67 Ebd.

68 Herbert Lüthy, Geschichte und Fortschritt, in: RudolfW. Meyer (Hrsg.), Das Problem des Fortschritts - heute, Darmstadt 1968, S. 6.

69 Wendorff, Zeit und Kultur, S. 217.

70 Ebd., S. 216-218. 
perspektiven entwickelt, wodurch sie zugleich als Indikator der Krisenhaftigkeit wie auch als deren Bearbeitungsmodus angesehen werden können"71. Das heißt, das Vanitas-Motiv war zugleich Ausdruck der ausgesprochenen Krisenhaftigkeit des Barock, speziell des 17. Jahrhunderts, als auch Form zur Kompensation dieses Krisenbewusstseins. Welche Formen eine barocke Auseinandersetzung mit dem Vanitas-Bewusstseins konkret annehmen konnte, zeigt der abschließende Blick in eine Auswahl exemplarischer zeitgenössischer Quellen.

\section{Der Blick in die zeitgenössischen Quellen}

Wie die bisherigen Ausführungen deutlich gemacht haben, kann das Vanitas-Motiv tatsächlich als Ausdruck einer überaus pessimistischen Welt- beziehungsweise Zukunftssicht innerhalb des barocken Zeitalters verstanden werden. Aufgrund der mannigfaltig vorhandenen Krisensymptome jener Zeit war das Bewusstsein darum, dass letztlich nur die Vergänglichkeit, das heißt der Tod, auf einen jeden wartete, geradezu allgegenwärtig. Zugleich fehlte es offenbar an der tröstlichen Vorstellung vom Übergang in ein unendliches Jenseits, wie sie etwa noch im Mittelalter präsent gewesen war, da sich das Zukunftsverständnis ebenfalls just zu jener Zeit in seinen Grundsätzen wandelte. Die Reaktion darauf war die bis dato umfassendste Konjunktur des Vanitas-Motives. Diese Symbolik irdischer Vergänglichkeit fand gerade im krisenbehafteten barocken Zeitalter großen Anklang, da die entsprechende kunst- und kulturgeschichtliche Ausgestaltung zugleich Ausdruck des allgegenwärtigen Krisenbewusstseins, aber auch Kompensationsmöglichkeit dieser Erfahrungen war. Einen Eindruck dessen vermitteln die drei folgenden Quellenbeispiele, die jeweils aus dem 17. Jahrhundert stammen.

\subsection{Cornelis Norbertus Gijsbrechts: "Memento mori” (1663)}

Im Original ist hier eine Abbildung der Vanitas-Stillleben: Memento mori, Cornelis Norbertus Gijsbrechts (1663).

Das Bild mit dem Titel "Memento mori"772 stammt vom flämischen Maler Cornelis Norbertus Gijsbrechts (auch Gysbrechts, 1657-1675). Die Signatur datiert das Bild in das Jahr 1663. Das Original, Öl auf Leinwand im Maß $147 \times 116 \mathrm{~cm}$, befindet sich heute unter der Inventarnummer 4770 in der Alten Pinakothek im Bestand der Bayerischen Staatsgemäldesammlungen in München. ${ }^{73}$

71 Nadine Hufnagel, Krise und Zukunft in Mittelalter und (Früher) Neuzeit, 31.10.2014-2.11.2014 Bayreuth, in: H-Soz-Kult, 8.10.2014, online [http://www.hsozkult.de/event/id/termine-26043], eingesehen 27.11.2015.

72 Konrad Renger, Cornelis Norbertus Gijsbrechts. Memento mori, 1663, in: Das flämische Stillleben. 1550-1680, Katalog zur Ausstellung des Kunsthistorischen Museums Wien und der Kulturstiftung Ruhr Hessen von 18.3.200221.7.2002, Lingen-Wien 2002, S. 49-50, Bild auf Seite 50. 
Auf einem „kostbaren orientalischen Teppich"74 sind mehrere Vergänglichkeitssymbole arrangiert: Im Zentrum befindet sich ein Totenschädel mit einem Ährenkranz, "dessen Körner als neue Saat aufgehen werden"75. Konrad Renger zufolge verweisen beide Symbole auf Auferstehung und ewiges Leben, ${ }^{76}$ zugleich ist der Totenkopf das offensichtlichste Vergänglichkeitsmotiv im dargestellten Stillleben. Umgeben ist dieser von einer geöffneten Taschenuhr im Vordergrund und einem liegenden Stundenglas in rechter Blickrichtung, jeweils Sinnbilder für die verrinnende Zeit. Des Weiteren zu sehen ist eine fast gänzlich heruntergebrannte Kerze, deren Flamme allerdings noch brennt, was als Ausdruck für das nahende Ende - gewissermaßen das baldige Erlöschen des ,Lebenslichtes' - interpretiert werden kann. Ergänzt wird dieses Ensemble von einer Geige mit Notenblatt, das mit den Worten "vanitas vanitatum extra Deum”, also „Ohne Gott Eitelkeit der Eitelkeit", beschriftet ist, während es sich bei den Noten um Ballette und Sarabande, also vergnügliche Stücke handelt. Gemeinsam mit dem prunkvollen Goldpokal im Hintergrund und dem prächtig blühenden Blumenstrauß stehen diese Symbole für die Vergänglichkeit irdischer Vergnügungen und Schönheit. Ebenso sind auch Besitz, Wissen und Ruhm vergänglich, worauf das im Hintergrund aufgeschlagene Buch und der Globus sowie die Malerpalette mit Pinseln und die im Vordergrund über die Tischkante hängende Proklamation mit der Überschrift „Von Gottes Gnaden, wir Albrecht in Ob ..." ${ }^{\prime 77}$ zeugen, die der Künstler offenbar öfter in seinen Bildern verwendete. ${ }^{78}$

Dieses Bild mit der stattlichen Sammlung seinerzeit gängiger Vanitas-Symbole und dem ernüchternden Titel Memento mori (Bedenke, dass du sterben wirst) steht demnach ganz im Sinne der Vergänglichkeitsmentalität des Barock. Tatsächlich handelt es sich dabei um ein eindrucksvolles Beispiel des Vanitas-Stilllebens, eines sich etwa seit den 1550er-Jahren etablierenden Genres, das gerade in der niederländischen Malerei zu einem eigenen Typus avancierte und seine Hochzeit im Zeitraum zwischen etwa 1550 und 1680 feierte. ${ }^{79}$ Hierbei ist zu erwähnen, dass Stillleben ab dem 16. Jahrhundert grundsätzlich „eigenständige Repräsentationsstücke von großer Sinnenfreude [waren], auf denen die Gegenstände des Alltags prunkvoll nobilitiert werden"80. Dass also selbst ein Genre, das sich der Darstellung von Alltagsgegenständen widmete, unter Verwendung des gesammelten Repertoires an Vergänglichkeitsmotiven zu einer eigenständigen, morbiden Kunstform aufstieg, kann allein schon als Beweis für das tiefe Vergänglichkeitsbewusstsein jener Zeit gewertet werden.

\footnotetext{
74 Renger, Gijsbrechts, S. 49.

75 Ebd.

76 Ebd.

77 Ebd

78 Ebd.

79 Ein Umstand, der wohl nicht zuletzt den Auswirkungen der Glaubenskriege und der noch jungen protestantischen Glaubensrichtung im niederländischen Raum zu schulden ist. Ewen, Vanitas, ENZ online.

80 Wilfried Seipel, Zum Geleit, in: Das flämische Stillleben. 1550-1680, Katalog zur Ausstellung des Kunsthistorischen Museums Wien und der Kulturstiftung Ruhr Hessen von 18.3.2002-21.7.2002, Lingen-Wien 2002, S. 9.
} 


\subsection{Andreas Gryphius: "Es ist alles gantz eytel" (1637/1663)}

Das Sonett ${ }^{81}$ „VANITAS, VANITATUM, ET OMNIA VANITAS. Es ist alles gantz eytel"82 ist eines der bekanntesten Barockgedichte und zugleich eines der bekanntesten lyrischen Produkte des „überragende[n] ${ }^{\prime 83}$ deutschen Barockdichters Andreas Gryphius (16161664). ${ }^{84}$ Unter dem erwähnten Titel erschien das vermutlich um 1636 entstandene Gedicht erstmals 1637 im sogenannten Lissaer Sonettbuch, der ersten Veröffentlichung deutscher Lyrik des Dichters, ${ }^{85}$ in folgendem Wortlaut:

„ICh seh' wohin ich seh / nur Eitelkeit auff Erden /

Was dieser heute bawt / reist jener morgen ein /

Wo itzt die Städte stehn so herrlich / hoch und fein /

Da wird in kurtzem gehn ein Hirt mit seinen Herden:

Der itzt so pocht und trotzt /laest ubrig Asch und Bein /

Nichts ist / daß auff der Welt koent unvergaenglich seyn /

Itzt scheint des Glueckes Sohn / bald donnerts mit beschwerden.

Der Thaten Herrligkeit muß wie ein Traum vergehn:

Solt denn die Wasserblaß / der leichte Mensch bestehn

Ach! was ist alles diß / was wir vor koestlich achten!

Alß schlechte Nichtigkeit? als hew / staub / asch und wind?

Als eine Wiesenblum / die man nicht wiederfind.

Noch wil / was ewig ist / kein einig Mensch betrachten!"86

Bereits der erste Vers gibt Hinweis auf Inhalt und Intention des Stückes, nämlich die „Eitelkeit auff Erden", die der Dichter in den folgenden Versen schrittweise erläutert. Der Lyriker beschreibt, wie einzelne Bauten und ganze Städte, Menschen und Tiere, aber auch Abstrakta wie das Glück und "der Thaten Herrligkeit" ${ }^{48}$ letztlich nichtig sind und vergehen, $d a$ "[n]ichts ist / daß auff der Welt koent unvergaenglich seyn" ${ }^{\prime 29}$. Auf unterschiedlicher Ebene verweist das Sonett also auf die Vergänglichkeit alles Irdischen, während etwa die Formulierung vom Traumgebilde oder von der Wasserblase auf ein gewisses Momentempfinden hinsichtlich der Existenz des Menschen und der Dinge hinweist.

In höchstem Maße interessant ist dabei, dass Gryphius zur Zeit der Entstehung dieses Gedichtes erst knapp 20 Jahre alt war. Ein vergleichsweise junges Alter für ein derart le-

81 Eine spezielle Gedichtform, die gerade im Zuge der Barocklyrik eine Hochzeit erlebte. Volker Meid, Barocklyrik (Sammlung Metzler 227), Stuttgart 2008², S. 70-72.

82 Andreas Gryphius: VANITAS, VANITATUM, ET OMNIA VANITAS. Es ist alles gantz eytel. Eccl. 1 v. 2., in: Ulrich Maché/ Volker Meid (Hrsg.), Gedichte des Barock (Reclam Universal-Bibliothek 9975[5]), Stuttgart 1980, S. 114

83 Borries/Borries, Deutsche Literaturgeschichte, S. 357.

84 Ebd.

85 Eberhard Mannack, Andreas Gryphius (Sammlung Metzler. Realien zur Literatur 76), Stuttgart 1986², 38-39.; Peter Nusser, Deutsche Literatur. Eine Sozial- und Kulturgeschichte. Band 2. Vom Barock bis zur Gegenwart, Darmstadt 2012, S. 101.

86 Andreas Gryphius, Es ist alles gantz eytel.

87 Ebd.

88 Ebd.

89 Ebd 
bensresignierendes und ernstes lyrisches Stück. Jens Ewen führt „Es ist alles eitel"90, die überarbeitete Version des Sonetts aus dem Jahre 1663,91 geradezu als Paradebeispiel für Vanitas-Darstellungen in der barocken Lyrik an. Der Historiker wertet das genannte Beispiel zugleich als Ausdruck einer "generellen Verunsicherung"92, die der Barockdichter Gryphius „unter dem Eindruck des Dreißigjährigen Krieges (1618-1648) und mit dem Aufkommen des kopernikanischen Weltbildes [...] erlebt[e]" ${ }^{193}$ haben soll.94

Eine These, die allerdings mit Vorbehalt zu betrachten ist. Denn obgleich der Schlesier Andreas Gryphius tatsächlich Zeitzeuge der verheerenden Glaubenskriege und des menschlichen Elends seiner eigenen Zeit war, ${ }^{95}$ das er gewiss auch in seinen Werken verarbeitete, darf die Barocklyrik im Allgemeinen als auch die Verarbeitung von Vanitas-Motiven im Speziellen nicht überbewertet werden: „Lyrik im Barock ist strenge Formkunst." ${ }^{\prime 96}$ Ziel war dabei nicht die Darlegung persönlicher Stimmungen und Erlebnisse, sondern die Wiedergabe allgemeingültiger Aussagen, die gewissermaßen nach allen Regeln der Kunst - man denke etwa an die Regelpoetik des Martin Opitz „ıdie Regeln zum Erlernen der Dichtkunst aufstellte und Musterbeispiele zur Nachahmung empfahl"97 - so eindringlich als möglich vermittelt werden sollten. ${ }^{98}$ Aus eben dieser regelhaften Kunstfertigkeit leitet sich auch die wiederkehrende Verwendung bekannter Motive, wie etwa der Vanitas, in den Werken zahlreicher Barockautoren ab. Wobei etwa Erika und Ernst von Borries festhalten, dass Themen wie die irdische Vergänglichkeit zugleich doch auch Ausdruck der „tiefen Religiosität des barocken Menschen [war], die gerade aus seiner äußeren Bedrohung verständlich wird"99.

Es ist alles gantz eytel steht damit also in mehrerlei Hinsicht Pate für das augenscheinliche Vergänglichkeitsbewusstsein und die Lebensresignation des barocken Zeitalters: Zum ersten als kunstfertige Verarbeitung eines seinerzeit populären Themas, zum

90 Andreas Gryphius: Es ist alles Eitel., in: Ulrich Maché/Volker Meid (Hrsg.), Gedichte des Barock (Reclam UniversalBibliothek 9975[5]), Stuttgart 1980, S. 114. Die adaptierte Version des bedeutend älteren Andreas Gryphius aus dem Jahre 1663 lautet:

"Was diser heute baut/ reist jener morgen ein:

Wo itzund Staedte stehn / wird eine Wisen seyn /

Auff der ein Schaefers-Kind wird spilen mit den Herden:

Was itzund praechtig blueht/sol bald zutretten werden.

Was itzt so pocht und trotzt ist Morgen Asch und Bein /

Nichts ist / das ewig sey / kein Ertz / kein Marmorstein.

Itzt lacht das Glueck uns an / bald donnern die Beschwerden.

Der hohen Thaten Ruhm muß wie ein Traum vergehn.

Soll denn das Spil der Zeit/ der leichte Mensch bestehn?

Ach! was ist alles diß / was wir koestlich achten /

Als schlechte Nichtikeit/als Schatten / Staub und Wind;

Als eine Wisen-Blum / die man nicht wider find't.

Noch wil was Ewig ist kein einig Mensch betrachten!"

91 Ebd.

92 Ewen, Vanitas, ENZ online.

93 Ebd.

94 Ebd.

95 Borries/Borries, Deutsche Literaturgeschichte, S. 357.

96 Ebd

97 Borries/Borries, Deutsche Literaturgeschichte, S. 356.

98 Ebd, S. 356-358.

99 Ebd, S. 356-359; Nusser, Deutsche Literatur, S. 101-103. 
zweiten als Ausdruck der unsicheren, lebensbedrohlichen Zeitumstände, zum dritten als Ausdruck der persönlichen Eindrücke und Erfahrungen des Zeitzeugen Andreas Gryphius, dem als Sohn eines Geistlichen zweifelsohne auch die tiefe Religiosität der Zeit inne wohnte. ${ }^{100}$

\subsection{Christian Hofmann von Hofmannswaldau: Vergaenglichkeit der schoenheit (1695)}

Da das Vanitas-Motiv, wie schon erläutert, ein überaus populäres Thema der barocken Dichtkunst war, das von nahezu allen Dichtern der Zeit aufgegriffen wurde, verwundert es kaum, dass aus der Feder Christian Hoffmanns von Hoffmannswaldau (16161679), einem Zeitgenossen Andreas Gryphius'101, ein weiteres, ähnlich geartetes Stück stammt: „Sonnet. Vergaenglichkeit der schoenheit"102 ist ein ebenfalls mit dem Motiv der Vanitas spielendes Klinggedicht, das 1695, und somit erst posthum, erschien.

„ES wird der bleiche todt mit seiner kalten hand

Dir endlich [Lesbia] mit der zeit umb deine brueste streichen /

Der liebliche corall der lippen wird verbleichen;

Der schultern warmer schnee wird werden kalter sand /

Der augen suesser blitz / die kraeffte deiner hand /

Fuer welchen solches faellt / die werden zeitlich weichen /

Das haar / das itzund kan des goldes glantz erreichen /

Tilgt endlich tag und jahr als ein gemeines band.

Der wohlgesetzte fuß / die lieblichen gebaerden /

Die werden theils zu Staub / theils nichts und nichtig werden /

Denn opfert keiner mehr der gottheit deiner pracht.

Diß und noch mehr als diß muß endlich untergehen /

Dieweil es die natur aus diamant gemacht."103

Wo Gryphius in seiner Erstversion von "Es ist alles gantz eytel" ein lyrisches Ich seine Gedanken zur allgegenwärtigen Vergänglichkeit mitteilen ließ, scheint das lyrische Ich in "Vergänglichkeit der Schönheit" ein lyrisches Du oder aber das Leserpublikum anzusprechen. Der Schein trügt in diesem Fall. Denn tatsächlich handelt es sich bei der vorliegenden Version wiederum um eine geringfügig adaptierte Variante des ursprünglichen Sonetts, in welchem in Vers zwei Lesbia anstelle von endlich zu lesen war.

100 Wolfgang Monath, Gryphius, Andreas, in: Neue Deutsche Biographie, online [http://www.deutsche-biographie. de/pnd118543032.html], eingesehen 29.11.2015. Monath führt hier weiter aus: „G[ryphius], dem jüngsten Kind aus der 3. Ehe seines Vaters, war tiefer Ernst, ja Schwermut wesenseigen. Schicksalsschläge, Verlust und Bedrängnis, die er von Jugend auf in überreichem Maße an sich und seiner Umwelt erlebte, mögen dazu die Erfahrung von der "Vergänglichkeit menschlicher Sachen" fest in sein Weltbild eingeprägt haben. Häufige Erkrankungen mit der Drohung des Todes verdüsterten sein Leben; früh verlor er Vater und Mutter; von den 6 Kindern aus der 2. Ehe seines Stiefvaters hat keines die Geburt lange überlebt (ähnlich starben auch die meisten seiner eigenen Kinder früh, eine Tochter blieb vom 4. Lebensjahr an physisch und geistig verkümmert); er war Zeuge von Pestepidemien und Feuersbrünsten, die Landstriche und Städte verheerten, erlebte aus nächster Nähe Leiden und Zerstörung des 30jährigen Krieges und litt unter der konfessionellen Verfolgung, die den Stiefvater und ihn selbst 1628 aus seiner Heimatstadt vertrieb."

101 Borries/Borries, Deutsche Literaturgeschichte, S. 359-360.

102 Christian Hoffmann von Hoffmannswaldau: Sonnet. Vergaenglichkeit der schoenheit, in: Ulrich Maché/Volker Meid (Hrsg.), Gedichte des Barock (Reclam Universal-Bibliothek 9975[5]), Stuttgart 1980, S. 274. 
Eine Tatsache, die „dem Gedicht eine wichtige Nuance nimmt"104. Mit dem Namen war, für die späthumanistisch gebildete Leserschaft klar, die schöne Konsulsgattin aus den Gedichten des römischen Dichters Catull gemeint, die ihn das Lieben und Leiden gelehrt hatte. „Ihr Name in einem Barockgedicht ist also Programm: er steht für erotische Reize und für kaltes Nicht-Erhören"105, auf das der Autor sozusagen reagierte, indem er der lyrischen Frauenfigur die eigene Vergänglichkeit vor Augen führte.

In diesem Sinne geht von Hoffmannswaldau in seinem Sonett weitaus spielerischer als etwa sein Zeitgenosse Gryphius mit dem Vanitas-Motiv um, indem er, vergleichbar der mittelalterlichen Minnelyrik, eine geradezu erotische Beziehung zwischen lyrischem Du respektive seiner Lesbia und dem teils personifizierten Tod aufleben lässt. Quasi selbst zum Leben erweckt, zeugt das Gedicht vom Tod, der mit "seiner kalten hand"106 der Schönen nach und nach ihre Reize nehmen wird. Anders als Gryphius, der die Endlichkeit alles Irdischen, die Vergänglichkeit von Mensch, Tier, Ding und Idee, verarbeitete, ist es bei von Hoffmannswaldau speziell die menschliche, oder konkreter die weibliche Schönheit, die letztlich „nichts und nichtig"107 und von keinerlei Wert mehr sein wird. Was allein über alle körperliche Vergänglichkeit erhalten bleibt, ist das "Hertze", ${ }^{108}$ das einem „diamant"109 gleich rein und (deshalb?) von Bestand sein wird. Also letztlich das Innerste eines Menschen, seine Seele oder aber die Erinnerung an denselben.

Wenngleich Christian Hoffmann von Hoffmannswaldau ein Zeitgenosse von Andreas Gryphius war und demnach von ähnlichen Erfahrungen und zeitgenössischen Einsichten geprägt gewesen sein dürfte, so scheint sein Sonett doch weitaus mehr eine rein lyrische Verarbeitung eines populären Themas darzustellen, als dies etwa bei Gryphius' Es ist alles gantz eytel der Fall ist. Weitaus verspielter und mit einem dem Barock nachgesagten Hang zum Ungewöhnlichen spielt von Hoffmannswaldau geradezu weltmännisch ${ }^{110}$ mit dem barocken Vergänglichkeits-Motiv. So ist er, anders als beispielsweise Gryphius, „,der, alle Mittel der poetischen Rhetorik perfekt beherrschend, immer den Ernst seines Anliegens spürbar werden ließ"111, dem Manierismus zuzuordnen. Das heißt, im Gegensatz zum weitaus ernsteren Schaffen seines Zeitgenossen, äuBerte sich von Hoffmannswaldaus Können in der künstlerischen Auslotung der gegebenen Kunstformen bis hin zum Selbstzweck, also der Manier. ${ }^{12}$ Dennoch kann auch der weltmännisch-spielerische Umgang mit dem Motiv der Vanitas als Ausdruck eines starken Vergänglichkeitsbewusstseins selbst in den lebensbejahenden, weltmännischen Kreisen gewertet werden, wenn man so will.

\footnotetext{
104 Borries/Borries, Deutsche Literaturgeschichte, S. 361.

105 Ebd, S. 360-361.

106 von Hoffmannswaldau, Vergaenglichkeit der schoenheit.

107 Ebd.

$108 \mathrm{Ebd}$

109 Ebd.

110 „Als Sohn eines kaiserlichen Rats geboren und in bedeutender öffentlicher Stellung, konnte er selbstverständlich die Haltung des überlegenen Weltmannes einnehmen." Borries/Borries, Deutsche Literaturgeschichte, S. 359; Erwin Rotermund,'Hofmann von Hofmannswaldau, Christian', in: Neue Deutsche Biographie, online [http://www. deutsche-biographie.de/pnd118706187.html], eingesehen 29.11.2015.

111 Borries/Borries, Deutsche Literaturgeschichte, S. 360.

112 Ebd., S. 359-361; Nusser, Deutsche Literatur, S. 107-109.
} 


\section{Schluss}

Was lässt sich abschließend also festhalten? Wie die exemplarisch vorgestellten Quellenbeispiele aus Kunst und Literatur gezeigt haben, war das Vergänglichkeits-Motiv zur Zeit des Barock und gerade im 17. Jahrhundert ein überaus populäres und beliebtes. Doch ist dieser Umstand allein schon Beweis genug für ein tatsächlich existentes Vergänglichkeitsbewusstsein innerhalb der barocken Gesellschaft? Zur Beantwortung dieser Frage sei eine Revue der im Zuge dieser Arbeit aufgestellten Erkenntnisse erlaubt:

1. Die Zukunft ist die Zeit, die noch kommt, und ist je nach räumlichen, zeitlichen und sozialen Bedingungen mit bestimmten Erwartungshaltungen verbunden. Das heißt, die Zukunft beziehungsweise die jeweilige Vorstellung davon ist keine anthropologische Konstante, sondern eine spezifische Denkform, ein menschliches Gedankenkonstrukt. Das, was jeweils in die Zukunft hineinprojiziert wird, ist also Summe der jeweiligen Raum-Zeit-Mensch-Bedingungen.

2. Im Laufe der Zeit herrschten dabei ganz unterschiedliche Vorstellungen von der Zukunft vor, die sich im abendländischen Bewusstsein etwa seit der Zeit des Frühen Mittelalters ausdifferenzierten und gerade im Zuge der auch hier untersuchten Frühen Neuzeit einen entscheidenden Wandel durchmachten: Es ist dies der Übergang vom geschlossenen, auf das Weltenende hin ausgerichteten Zukunftsbegriff hin zur Entdeckung der Zukunft als offenem und weitem Zeithorizont. Ein mentalitätsgeschichtlicher Wandlungsprozess, der sich schon im 16. Jahrhundert manifestierte, der die eschatologische Zukunftssicht aber erst im 18. Jahrhundert flächendeckend ablöste.

3. Etwa zur selben Zeit erlebte das Vanitas-Motiv, ein seit dem Altertum bekannter Topos, der in teils eindeutig morbiden Bildern die Vergänglichkeit alles Irdischen thematisiert, seine bis dato größte Blütezeit.

4. Das Barock, also die Zeit vom späten 16. bis zum späten 18. Jahrhundert, gilt grundsätzlich als Zeitalter des Wandels, der Gegensätze und Spannungen beziehungsweise wird aus unterschiedlicher Perspektive als Krisenzeitalter diskutiert. In diesem Sinne war die barocke Gesellschaft, insbesondere die des 17. Jahrhunderts, in vielerlei Hinsicht von einer umfassenden Erfahrung der Unsicherheit geprägt, zu der zweifelsohne auch der tiefgreifende Wandel des abendländischen Zeit- respektive Zukunftsbewusstseins zu zählen ist, der sich in eben diesem Zeitfenster vollzog. Als signifikantestes Krisensymptom dieser Zeit kann dabei fraglos der Dreißigjährige Krieg festgemacht werden, der in Kombination mit klimatischen Veränderungen, Krankheitswellen und Hungersnöten das Elend der Menschen empfindlich verstärkte und die Zahl der Todesopfer nahezu vervierfachte. Der Tod, die eigene Vergänglichkeit, war also in der Tat allgegenwärtig.

Unter diesen Gesichtspunkten betrachtet darf das Motiv der Vanitas (Vergänglichkeit) für die Zeit des Barock also mit einiger Bestimmtheit als weit mehr denn als rein künstlerische Ausdrucksform gewertet werden, wenngleich die exemplarische Quellenana- 
lyse gezeigt hat, dass zumindest die barocke Lyrik in erster Form Kunst war. Dennoch verdeutlicht allein der Umstand, dass das Vanitas-Motiv seinerzeit so populär war und in ernsten ebenso wie in spielerischen literarischen Stücken Verarbeitung fand und in der Malerei sogar zur eigenen Kunstgattung - dem Vanitas-Stillleben - avancierte, wie tiefgreifend das allgemeine Vergänglichkeitsbewusstsein tatsächlich gewesen sein muss. Oder um noch einmal die Worte Herbert Lüthys aufzugreifen: Die „Katastrophenzeit des 17. Jahrhunderts war nicht dazu angetan, einen Geschichtsoptimismus hervorzubringen; die Geschichtsschau des Barock ist die Geschichte als Trauerspiel“113.

Die dieser Arbeit zugrundeliegende Forschungsfrage, ob denn das Vanitas-Motiv im Barock Ausdruck für einen grundsätzlichen Zukunftspessimismus innerhalb der eigentlich stets positiv konnotierten Frühen Neuzeit war, lässt sich demnach mit einem „Ja mit Vorbehalt" beantworten. Denn freilich ist weder die exemplarische Analyse eines einzelnen Motives noch die eines einzigen Epochenausschnittes ausreichend, um das Zukunftsempfinden einer ganzen Epoche auszumachen. Um der frühneuzeitlichen Zukunftsmentalität nachzuspüren, bedürfte es umfangreicher weiterer Untersuchungen, wobei weitere Einzelstudien womöglich zu einem ganz anders gearteten Bild der barocken beziehungsweise frühneuzeitlichen Zukunftsvorstellung(en) gelangten. Dennoch lässt sich anhand der hier vorgenommenen Untersuchung für die Zeit des Barock, wenigstens aber für das 17. Jahrhundert, eindeutig eine von Vergänglichkeit, Nichtigkeit und dem nahenden Tod geprägte Zukunftssicht festhalten, die sich in Form des auf Hochkonjunktur befindlichen Vanitas-Motives in Kunst und Literatur äußerte und die sich umso mehr erhärtet, je weiter man den mannigfachen Krisensymptomen jener Zeit nachspürt.

\section{Quellen}

Andreas Gryphius: Es ist alles Eitel., in: Maché, Ulrich/Meid, Volker (Hrsg.), Gedichte des Barock (Reclam Universal-Bibliothek 9975[5]), Stuttgart 1980, S. 114.

Andreas Gryphius: VANITAS, VANITATUM, ET OMNIA VANITAS. Es ist alles gantz eytel. Eccl. 1 v. 2., in: Maché, Ulrich/Meid, Volker (Hrsg.), Gedichte des Barock (Reclam Universal-Bibliothek 9975[5]), Stuttgart 1980, S. 114.

Christian Hoffmann von Hoffmannswaldau: Sonnet. Vergaenglichkeit der schoenheit, in: Maché, Ulrich/Meid, Volker (Hrsg.), Gedichte des Barock (Reclam Universal-Bibliothek 9975[5]), Stuttgart 1980, S. 274.

Cornelis Norbertus Gijsbrechts, Memento mori, 1663, Öl auf Leinwand, Inv. Nr. 4770, Alte Pinakothek, Bayerische Staatsgemäldesammlungen, München.

Georg Wilhelm Friedrich Hegel: Vorlesungen über die Philosophie der Geschichte, in: Georg Wilhelm Friedrich Hegel, Werke in zwanzig Bänden, Eva Moldenhauer und Karl Markus Michel (Hrsg.), Band 17, Frankfurt am Main 1969. 


\section{Literatur}

Ariès, Philippe, Geschiche des Todes, München 1995².

Borries von, Erika/Borries von, Ernst, Deutsche Literaturgeschichte. Band 1. Mittelalter Humanismus - Reforrmationszeit - Barock, München $2006^{5}$.

Burbaum, Sabine, Barock (Kunst-Epochen 8; Reclams Universal-Bibliothek 18175), Stuttgart 2003.

Dinzelbacher, Peter, Zu Theorie und Praxis der Mentalitätsgeschichte, in: Dinzelbacher, Peter (Hrsg.), Europäische Mentalitätsgeschichte. Hauptthemen in Einzeldarstellungen, Stuttgart 1993, S. XV-XXXVII.

Ewen, Jens, ,Vanitas', in: Enzyklopädie der Neuzeit, online [http://referenceworks.brillonline.com/entries/enzyklopaedie-der-neuzeit/vanitas-a4511000], eingesehen 19.11.2015.

Gerber, Doris, Was heißt, vergangene Zukunft'? Über die zeitliche Dimension der Geschichte und die geschichtliche Dimension der Zeit, in: Geschichte und Gesellschaft 32 (2006), Heft 2, S. 176-200.

Geuenich, Dieter, Zukunftsvorstellungen im Mittelalter. Antrittsvorlesung an der Universität - GH - Duisburg am 30. Mai 1989, online [http://www.univie.ac.at/igl.geschichte/zeller/ws_2010_11/materialien_ku/Geuenich.pdf], eingesehen 16.11.2015.

Götze, Lutz, Zeitkulturen. Gedanken über die Zeit in den Kulturen (Im Medium fremder Sprachen und Kulturen 6), Frankfurt am Main 2004

Hölscher, Lucian, Die Entdeckung der Zukunft (Europäische Geschichte), Frankfurt am Main 1999.

Hufnagel, Nadine, Krise und Zukunft in Mittelalter und (Früher) Neuzeit, 31.10.2014 2.11.2014 Bayreuth, in: H-Soz-Kult, 8.10.2014, online [http://www.hsozkult.de/event/id/ termine-26043], eingesehen 27.11.2015.

Ingen van, Ferdinand, Vanitas und Memento mori in der deutschen Barocklyrik, Groningen 1966.

Koselleck, Reinhart, Vergangene Zukunft. Zur Semantik geschichtlicher Zeiten (suhrkamp taschenbuch wissenschaft 757), Frankfurt am Main 1979.

Kreibich, Rolf, Zukunftsforschung. ArbeitsBericht Nr. 23/2006, IZT, Berlin 2006, S. 3, online [https://www.izt.de/fileadmin/downloads/pdf/IZT_AB23.pdf], eingesehen 1.10.2015.

Luhmann, Niklas, The Future Cannot Begin: Temporal Structures in Modern Society, in: Social Research, 43 (1976), Nr. 1, S. 130-152.

Lüthy, Herbert, Geschichte und Fortschritt, in: Meyer, RudolfW. (Hrsg.), Das Problem des Fortschritts - heute, Darmstadt 1968.

Mader, Eric-Oliver, Zeiterfahrung, in: Enzyklopädie der Neuzeit, online [http://referenceworks.brillonline.com/entries/enzyklopaedie-der-neuzeit/zeiterfahrung-a4858000], eingesehen 4.11.2015. 
Mannack, Eberhard, Andreas Gryphius (Sammlung Metzler. Realien zur Literatur 76), Stuttgart $1986^{2}$.

Meid, Volker, Barocklyrik (Sammlung Metzler 227), Stuttgart $2008^{2}$.

Minois, Georges, Geschichte der Zukunft. Orakel, Prophezeiungen, Utopien, Prognosen, Düsseldorf-Zürich 1998.

Monath, Wolfgang, ,Gryphius, Andreas', in: Neue Deutsche Biographie, online [http:// www.deutsche-biographie.de/pnd118543032.html], eingesehen 29.11.2015.

Nusser, Peter, Deutsche Literatur. Eine Sozial- und Kulturgeschichte. Band 2. Vom Barock bis zur Gegenwart, Darmstadt 2012.

Pfisterer, Ulrich, Barock. 1. Einleitung, in: Enzyklopädie der Neuzeit, online [http://referenceworks.brillonline.com/entries/enzyklopaedie-der-neuzeit/barock-a0349000], eingesehen 25.11.2015.

Renger, Konrad, Cornelis Norbertus Gijsbrechts. Memento mori, 1663, in: Das flämische Stillleben. 1550-1680, Katalog zur Ausstellung des Kunsthistorischen Museums Wien und der Kulturstiftung Ruhr Hessen von 18.3.2002-21.7.2002, Lingen-Wien 2002, S. 49-50.

Renger, Konrad, Vanitas-Stillleben, in: Das flämische Stillleben. 1550-1680, Katalog zur Ausstellung des Kunsthistorischen Museums Wien und der Kulturstiftung Ruhr Hessen von 18.3.2002-21.7.2002, Lingen-Wien 2002, S. 140-160.

Rotermund, Erwin, ,Hofmann von Hofmannswaldau, Christian', in: Neue Deutsche Biographie, online [http://www.deutsche-biographie.de/pnd118706187.html], eingesehen 29.11.2015.

Schmidt-Gernig, Alexander, Rezension zu: Hölscher, Lucian, Die Entdeckung der Zukunft, in: H-Soz-Kult 10.2.2000, online [http://hsozkult.geschichte.hu-berlin.de/rezensionen/252.pdf], eingesehen 16.9.2015.

Seipel, Wilfried, Zum Geleit, in: Das flämische Stillleben. 1550-1680, Katalog zur Ausstellung des Kunsthistorischen Museums Wien und der Kulturstiftung Ruhr Hessen von 18.3.2002-21.7.2002, Lingen-Wien 2002, S. 9.

Vanitas, in: Duden, online [http://www.duden.de/rechtschreibung/Vanitas], 19.11.2015. Vogler, Günter, Europas Aufbruch in die Neuzeit 1500-1650 (Handbuch der Geschichte Europas 5; UTB 2385), Stuttgart 2005.

Wendorff, Rudolf, Zeit und Kultur. Geschichte des Zeitbewußtseins in Europa, Opladen 1980.

Zukunft, in: Duden, online [http://www.duden.de/rechtschreibung/Zukunft], eingesehen 7.10.2015. 
Zukunft, in: Kluge, Friedrich, Etymologisches Wörterbuch der deutschen Sprache, Berlin-New York 201225, online [https://vpn.uibk.ac.at/+CSCO+1h756767633A2F2F6A 6A6A2E71727465686C6772652E70627A++/view/Kluge/kluge.12813?rskey=0fC8GB

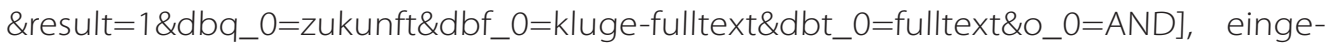
sehen 7.10.2015.

Lisa-Marie Gabriel ist Studentin der Geschichtswissenschaften (Masterstudium Geschichte und Alte Geschichte) im 2. Semester an der Universität Innsbruck.

Lisa-Marie.Gabriel@student.uibk.ac.at

\section{Zitation dieses Beitrages}

Lisa-Marie Gabriel, Zukunftspessimismus in der Frühen Neuzeit? Überlegungen zu frühneuzeitlichen Zukunftsvorstellungen am Beispiel des Vanitas-Motives zur Zeit des Barock, in: historia.scribere 9 (2017), S. 73-93, [http://historia.scribere.at], 2016-2017, eingesehen 14.6.2017 (=aktuelles Datum).

(C) Creative Commons Licences 3.0 Österreich unter Wahrung der Urheberrechte der Autorlnnen. 
